\title{
Reactive 0xygen Species Regulate Innate But Not Adaptive Inflammation in ZAP70-Mutated SKG Arthritic Mice
}

\author{
Simon Guerard, Rikard Holmdahl, and Kajsa Wing
}

From the Division of Medical Inflammation Research, Department of Medical Biochemistry and Biophysics, Karolinska Institutet, Stockholm, Sweden

\author{
Accepted for publication \\ May 5, 2016. \\ Address correspondence to \\ Kajsa Wing, Ph.D., Division of \\ Medical Inflammation \\ Research, Department of \\ Medical Biochemistry and \\ Biophysics, Karolinska \\ Institute, Stockholm, \\ Sweden. E-mail: kajsa.wing@ \\ ki.se.
}

\begin{abstract}
Polysaccharides from Saccharomyces cerevisiae can induce arthritis, ileitis, and interstitial pneumonitis in BALB/c ZAP70 (W163C)-mutant (SKG) mice via T helper 17-cell-dependent pathways. However, little is known regarding the factors influencing disease severity. We investigated mannan-induced arthritis in SKG mice and how NADPH oxidase 2-derived reactive oxygen species (ROS) regulate disease. SKG mice were highly susceptible to both IL-17-mediated T-cell-driven arthritis and T-cell-independent acute psoriasis-like dermatitis. In vivo imaging revealed more ROS in joints of arthritic SKG mice compared to wild-type mice, which links ROS and joint inflammation. Still, ROS deficiency in SKG.Ncf1 ${ }^{m 1 j / m 1 j}$ mice greatly increased severity of arthritis and dermatitis, a difference that could not be attributed to increased T-cell activation, thymic selection, or antibody production. However, when ROS production was restored in $\mathrm{CD} 8^{+}$macrophages, inflammation reverted to baseline, demonstrating a regulatory role of macrophage-derived ROS in autoimmunity. Thus, arthritis in SKG mice is a useful model to study the role of ROS in innate-driven chronic inflammation independently of adaptive immunity. (Am J Pathol 2016, 186: 2353-2363; http://dx.doi.org/10.1016/j.ajpath.2016.05.014)
\end{abstract}

A key issue in immunology is to reveal the interplay of factors that lead to multifactorial diseases, such as rheumatoid arthritis and psoriasis. In SKG mice, altered thymic selection because of a ZAP70 mutation predisposes mice to arthritis after challenge with polysaccharides from Saccharomyces cerevisiae. The T-cell dependency of this model is well characterized but less is known about what influences disease severity and how other cell populations contribute. NADPH oxidase 2 (NOX2)-derived reactive oxygen species (ROS) produced by phagocytes are suggested to prevent collagen-induced arthritis via T-cell regulation. To further investigate ROS in T-cell-driven autoimmunity, we introduced the $N c f 1^{m 1 j / m 1 j}$ mutation in C57BL/10.Q SKG mice to prevent ROS burst.

ROS produced by neutrophils and macrophages are antimicrobial agents essential for host defense. ${ }^{1}$ Lack of ROS burst in humans with genetic defects in the NOX2 complex leads to chronic granulomatous disease, a rare immunodeficiency characterized by incapacity of phagocytes to kill bacteria and formation of granuloma in various organs. ${ }^{2}$ Increased ROS concentrations in chronic inflammation have, on the other hand, long been suspected to be associated with tissue damage, and thus contribute to autoimmune disease. ${ }^{3-5}$ Paradoxically, chronic granulomatous disease patients are predisposed to autoimmune diseases, which is not directly linked to increased infections. ${ }^{6,7}$ The theory that ROS are deleterious in autoimmunity was further questioned when a natural polymorphism in neutrophil cytosolic factor 1 ( Ncfl, alias $\mathrm{p} 47^{\text {phox }}$ ) that reduced NOX2-dependant ROS burst was found to increase arthritis susceptibility in rats. ${ }^{1,8}$ This was confirmed in $N c f 1^{m l j / m l j}$ and $G P 91^{-/-}$mice, which both lack a

Supported by Swedish Research Council grants 522-2009-2548 (K.W.) and 521-2010-2894 (R.H.); Åke Wibergs Stiftelse grants 367990049, 924563126, and 940219686 (K.W.); Pr. Nanna Svartz Stiftelse (K.W.); the Swedish Society of Medicine (K.W.); Ulla och Gustaf af Ugglas Stiftelse (K.W.); Swedish Rheumatic Association (K.W.); King Gustav V 80th Birthday Foundation (K.W. and R.H.); the Swedish Strategic Science Foundation (R.H.); Knut and Alice Wallenberg Foundation grant KAW2010.0148 (R.H.); the European Union Innovative Medicine Initiative (EU IMI) project BeTheCure and EU FP7 Neurinox projects (R.H.).

Disclosures: None declared. 
functional NOX2 complex, and are more susceptible to collagen-induced arthritis (CIA), a model of chronic arthritis in which joint-specific inflammation is triggered by immunization against the joint-specific protein collagen type II (CII). ${ }^{9,10}$

Studies aimed to understand the mechanism by which ROS protect against autoimmunity show that increase in reduced thiols on the surface of $\mathrm{T}$ cells in ROS-deficient rodents increase their activation status and arthritogenicity. ${ }^{11}$ It was also reported that ROS deficiency increases production of IL- 6 and transforming growth factor- $\beta$ by dendritic cell activated with Mycobacterium tuberculosis, which leads to an increased $\mathrm{T}$ helper 17 response and susceptibility to CIA. ${ }^{12}$ Moreover, T-cell tolerance toward endogenous CII was observed to be broken in $N c f 1^{m l J / m l J}$ mice. ${ }^{13}$ Whether this effect was central or peripheral remains an open question. Finally, ROS from macrophages have been suggested to be protective in CIA. ${ }^{2,14}$ Altogether, these findings indicate that ROS derived from APC have anti-inflammatory properties that operate by affecting T-cell pathogenicity. Still, anti-inflammatory ROS-mediated Tcell-independent mechanisms have also been suggested. ${ }^{15}$ For instance, ROS secreted by neutrophils contribute to the formation of neutrophil extracellular traps in inflamed tissue, which was recently shown to be anti-inflammatory by absorbing cytokines. ${ }^{16}$ Taken together, the exact mechanism by which ROS protect against autoimmunity remains to be elucidated.

To further investigate the role of ROS in inflammation and T-cell activation, we selected the T-cell-mediated chronic arthritis in SKG mice as a model. The SKG mice have a point mutation in ZAP7O that leads to lower binding affinity to ITAM motives of CD3- $\zeta$ and reduced TCR signal transduction. ${ }^{17}$ This results in a reduced thymic output and a skewed T-cell repertoire that, on activation of innate immunity with $\beta$-glucans such as zymosan and mannan, precipitates into autoimmunity predominantly against joints. $^{3-5,18,19}$ Although these mice spontaneously develop titers of autoantibodies such as anti-CII and rheumatoid factor, arthritis in SKG mice is shown to be a T-cell-driven IL17-mediated disease. ${ }^{7,17,20}$ The distinct T-cell dependency combined with critical need for innate activation suggests that this model is suitable to study the ROS-dependent interplay between innate and adaptive immunity. Interestingly, our results show a protective role for macrophage-derived ROS in arthritis that appears independent of T- and B-cell activation.

\section{Materials and Methods}

\section{Animal Experimentation}

Mice were bred and maintained under specific pathogen-free environment with 12 hours light/dark cycle and were provided food and water ad libitum. All animal procedures were approved by regional ethical committees at Karolinska Institute and were in accordance with European Union legislation for experimental animal welfare. Experiments were conducted with sex-/age-matched groups using littermate controls whenever possible, and otherwise cage controls. Investigators were blinded to mice genotype and treatment groups.

Mice

All mice in this study were on the C57BL/10.Q/rhd background expressing the $\mathrm{H} 2-\mathrm{A}^{\mathrm{q}}$ haplotype (referred to as $w t$ ). $Z A P 70^{\text {skg/skg }}$ mice (referred to as SKG), which were a kind gift from Shimon Sakaguchi (Kyoto University, Kyoto, Japan), were backcrossed more than 10 generation from BALB/C background to C57BL/10.Q/rhd.Ncf1 $1^{m 1 J / m 1 J}$ mutated mice (referred to as $N c f 1^{* / *}$ ), previously described, and were intercrossed with SKG mice to generate ROS-deficient ZAP7O ${ }^{\text {skg/skg }} . N c f 1^{m l J / m l J}$ (referred as SKG.Ncfl ${ }^{* / *}$ ). ${ }^{9}$ Transgenic mice expressing functional Ncf1 restricted under human $C D 68$ promoter previously described were further intercrossed with ZAP70 ${ }^{\text {skg/skg }} . N c f I^{m I J / m I J}$ to restore ROS capacity in macrophages (referred to as SKG.MN). ${ }^{9,10,14}$ B6.129P2-Tcrb ${ }^{\text {tmIMom }} / \mathrm{J}$ were acquired from Jackson Laboratory (Bar Harbor, ME) and backcrossed over 10 generations on the C57BL/10.Q/rhd background and intercrossed with $N c f I^{m I J / m I J}$ (respectively referred as $T C R \beta^{-/-}$and $\left.T C R \beta^{-/-} . N c f 1^{* * *}\right)$.

\section{Mannan-Induced Autoimmunity}

Male and female mice, aged 8 to 12 weeks old, were injected i.p. with $20 \mathrm{mg}$ of mannan extracted from S. cerevisiae (Sigma-Aldrich: batch 109K3780V and 061M7355). Joint and skin inflammation was then evaluated blindly every other day. A 60-point score for arthritis was used in which each inflamed toe and knuckle is given a score of 1 and wrist/ankle a score of 5 , as previously described. ${ }^{11,21}$ For skin inflammation, a 3-point scale described previously was used. ${ }^{12,22}$ Briefly, the score reflects the average severity of redness, pealing, and hair loss on limbs evaluated blindly ( 1 , weak; 2 , moderate; 3 , severe).

\section{In Vivo Imaging of ROS in Joints}

Production of ROS in joints was monitored in vivo using the bioluminescent probe L-012 [8-amino-5-chloro7-phenylpyrido(3,4-d)pyridazine-1,4(2H,3H)dione] purchased from Wako Chemical (Nordic Biolabs, Täby, Sweden) as previously described with some modifications. ${ }^{13,23}$ Briefly, mice were first sedated by i.p. injection of $50 \mathrm{mg} /$ $\mathrm{kg}$ ketamine and $1 \mathrm{mg} / \mathrm{kg}$ dormitor (Orion Corporation, Espoo, Finland). The probe L-012 was then injected i.p. (40 $\mathrm{mg} / \mathrm{kg}$ ) and mice were immediately imaged using PhotonIMAGER (Biospace Lab, Paris, France) for 20 minutes. Image acquisition and analysis were performed using M3vision software version 1.0.8 (Biospace Lab, Nesles la Vallée, France). Photon intensity was measured in hind paws and expressed in $\mathrm{ph} / \mathrm{s} / \mathrm{cm}^{2} / \mathrm{sr}$ to normalize by the area used for measurement. 


\section{Anti-Rat CII ELISA}

Blood from mice was taken by submandibular bleeding or by heart puncture on experimental end point. After coagulation, serum was separated by centrifugation and kept at $-20^{\circ} \mathrm{C}$. Plates were first coated overnight at $4^{\circ} \mathrm{C}$ with 10 $\mu \mathrm{g} / \mathrm{mL}$ of rat CII and then blocked using $2 \%$ bovine serum albumin. ${ }^{24}$ Diluted sera were then added overnight at $4{ }^{\circ} \mathrm{C}$ along with standardized sera to determine absolute concentrations. Antibodies binding CII were detected using horseradish peroxidase-conjugated anti-mouse IgGK (clone 187.1; SouthernBiotech, Birmingham, AL) and ABTS (Roche Diagnostics, Mannheim, Germany) as substrate. Absorbance was measured at $405 \mathrm{~nm}$ on a Synergy2 plate reader (BioTek Instruments, Winooski, VT).

\section{Serum Transfer}

Blood was obtained by heart puncture from wt, SKG, and SKG. $N c f 1^{* / *}$ mice 60 days after $20 \mathrm{mg}$ i.p. injection of mannan. After centrifugation, pooled sera were stored at $-20^{\circ} \mathrm{C}$ until needed. SKG mice were injected i.p. with $20 \mathrm{mg}$ of mannan and randomly attributed to receive $3 \times 200 \mu \mathrm{L}$ i.v. of pooled sera from wt, SKG, or SKG.Ncfi ${ }^{* / *}$ at day 19, 23, and 27 to mimic a slow gradual increase in antibody titers.

\section{Adoptive Cell Transfer}

Cells were first isolated from joint draining lymph nodes (dLN; axillary, brachial, and inguinal) by mechanical disruption from SKG and SKG.Ncf1 $1^{* / *}$ mice 5 days after mannan injection. Cells were pooled, and 10 million cells per mice were transferred i.v. to either $T C R \beta^{-/-}$or $T C R \beta^{-/-} . N c f 1^{* / *}$ recipients. After 3 weeks of repopulation by $\mathrm{CD}^{+}{ }^{+} \mathrm{T}$ cells, which was monitored in the blood by flow cytometry, disease was triggered in chimeric mice with $20 \mathrm{mg}$ i.p. of mannan.

\section{Flow Cytometry}

Single-cell suspensions were prepared by mechanical disruption of dLN (axillary, brachial, and inguinal), spleen, and thymi, or after lysis of erythrocyte from heparinized peripheral blood. After Fc $\gamma$ R-blockade by incubating cells 5 minutes at $4^{\circ} \mathrm{C}$ with $2.4 \mathrm{G} 2$ antibody, extracellular antigens were stained 20 minutes at $4^{\circ} \mathrm{C}$ in phosphate-buffered saline with $1 \%$ fetal bovine serum. Cells were also fixed and permeabilized using Foxp3 Fix/Perm buffer set (BioLegend) and stained for intracellular antigens and cytokines when applicable. Samples were acquired using BD LSR II flow cytometer operated by Diva software version 8.0.1 (BD Biosciences, San Jose, CA), and the data were analyzed using FlowJo software version 10.1 (TreeStar, Inc., Ashland, OR).

To identify cell populations in peripheral blood, cells were stained with anti-CD45-PE-Cy7 (30-F11), anti-CD3APC (145-2C11), anti-CD4-PB (RM4-5), anti-CD8aFITC (53-6.7), anti-CD19-Alexa Fluor 700 (6D5),
anti-TCRß-PerCP-Cy5.5 (H57-597), or anti-TCR $\gamma \delta$-PE (GL3). The development stage of thymocytes was evaluated by staining cells isolated from thymi of 21-day-old mice with anti-CD4-PB, anti-CD8a-FITC, anti-CD25-PE-Cy7 (PC61), anti-CD44-APC (IM7), and anti-TCR $\beta$-PerCPCy5.5. For T-cell-activation markers, cells were stained with anti-CD3-PerCP-Cy5.5, anti-CD4-PB, anti-CD25APC, and anti-CD62L-PE-Cy7 (MEL-14) followed by anti-Ki-67-PE (MOPC-21) and Foxp3-FITC (FJK-16S) after fixation/permeabilization. For cytokine production staining, 2 million cells were stimulated in vitro with $50 \mathrm{ng} / \mathrm{mL}$ phorbol 12-myristate 13-acetate, $1 \mu \mathrm{mol} / \mathrm{L}$ of ionomycin, and $10 \mu \mathrm{g} / \mathrm{mL}$ brefeldin $\mathrm{A}$ for 4 hours at $37^{\circ} \mathrm{C}$ in Dulbecco's modified Eagle's medium supplemented with $10 \%$ fetal bovine serum. Cells were then stained with anti-CD3-APC, anti-CD4-Qdot 605, anti-CD45R-FITC, anti-HC class II-FITC (2G9), CD49b-FITC (DX5), CD11b-FITC (M1/70), followed by anti-IL-17A-PB (TC11-18H10.1) and anti-IFN $\gamma$-PECy7 (XMG1.2). For all staining, dead cells were excluded using LIVE/DEAD Fixable Near-IR Dead (Life Technologies). Cell debris were excluded by forward and side scattering, whereas doublets were by comparing FSC-A and FSC-H. All antibodies were from Becton Dickinson (San Diego, CA), eBioscience (San Jose, CA), or BioLegend (San Jose, CA).

\section{Joint Histology}

Hind paws were fixed in paraformaldehyde, decalcified in EDTA, and embedded in paraffin as previously described. ${ }^{17,22}$ Sections were stained with hematoxylin and eosin, and inflammation was scored on a 30-point scale. Points were attributed as follows: synovium hyperplasia (2), infiltration (2), angiogenesis (2), pannus formation (2), necrosis (2), cartilage erosion (2) or new formation (2), bone erosion (2) or new formation (2), joint structure destruction (6), and ankylosis (6).

\section{Statistical Analysis}

Statistical analyses were performed using GraphPad Prism5 software version 6.07 (La Jolla, CA). One-way analysis of variance with Tukey post hoc test were used to compare group for in vivo ROS production, antibody concentrations, and flow cytometry parameters. Two-way analysis of variance with Bonferroni post hoc test were used to compare groups for clinical score of joints and skin inflammation. Values are expressed as means \pm SEM. Results are considered significant for $P<0.05$.

\section{Results}

ROS Deficiency Worsens Autoimmunity in SKG Mice

To investigate the role of ROS in SKG mice, they were backcrossed from $\mathrm{BALB} / \mathrm{C}$ to $\mathrm{C} 57 \mathrm{BL} / 10 . \mathrm{Q} / \mathrm{rhd}$ 
background and intercrossed with ROS deficient $N c f 1^{m I J /}$ ${ }^{m 1 J}$ mice. ${ }^{25} \mathrm{We}$ found that a single i.p. injection of mannan resulted in an acute joint and skin inflammation that peaked around day 7 with a disease incidence close to $100 \%$ (Figure 1, A-D). The inflammation resolved in both wt and $N c f 1^{* * *}$ mutated mice after 10 to 14 days. However, in mice homozygote for the ZAP70 skg mutation (SKG and SKG. $N c f 1^{* * *}$ ), the arthritis stabilized and became chronic. Interestingly, lack of functional NOX2 complex in SKG.Ncf1 ${ }^{* * *}$ mice leads to a more severe disease phenotype for both arthritis and psoriasis-like dermatitis (Figure 1, A and C). In particular, the chronic phase is significantly more severe in SKG.Ncf1 $1^{* * *}$ mice compared with SKG mice $(P<0.001)$. Although SKG mice still have a few red and slightly swollen joints, in SKG. $N c f 1^{* / *}$ mice both the number of joints and the severity of inflammation is greatly increased. The psoriasis-like dermatitis is selflimited and the severity is mainly affected by the $N c f I$ mutation (wt versus $N c f 1^{* / *} P<0.001$ from day 4 to 7 ; SKG versus SKG.Ncfl ${ }^{* / *} P<0.001$ from day 4 to 10 ), which support previous results that mannan-induced psoriasis-like dermatitis is dependent on ROS but independent of adaptive immunity, in particular $\alpha \beta T$ cells. ${ }^{22}$ However, the acute phase is also slightly affected by the ZAP7O mutation (wt versus SKG $P<0.05$ on day 7 ; $N c f 1^{* / *}$ versus SKG. $N c f 1^{* / *} P<0.01$ from day 6 to 10 ), which
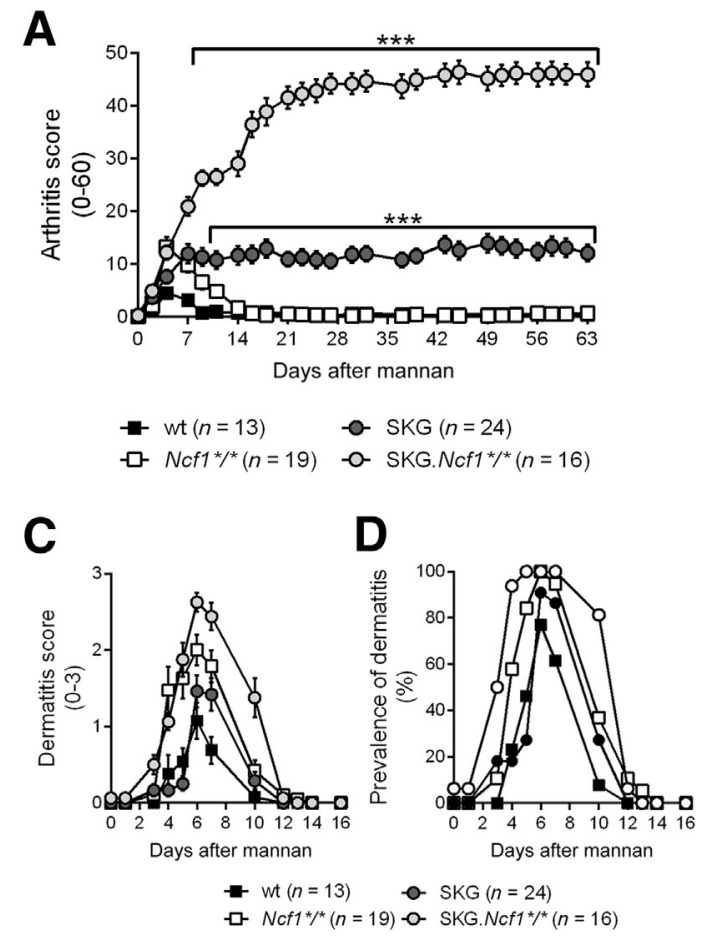

indicates that skin inflammation and joint inflammation overlaps in time in SKG mice.

Joint Inflammation in SKG Mice Is Associated with a Local ROS Production

Because ROS production by phagocytes in tissue is usually associated with inflammation, we investigated the ROS response in vivo using the bioluminescence probe L-012 after mannan injection in the respective groups (Figure 1E and Supplemental Figure S1). Although there was no detectable difference between naïve wt and SKG mice (day 0 ), SKG mice were shown to have higher ROS production in joints both in the acute (day 7 and 11) and chronic phase (day 60) (Figure 1E). As expected, neither $N c f 1^{* * *}$ nor SKG.NCf1 ${ }^{* * *}$ mice had detectable levels of ROS in joints after mannan injection despite being susceptible to autoimmunity.

The ROS signal detected in joints during the acute phase could also partly originate from the skin inflammation; because of technical limitations, we cannot distinguish between the two sources of signal. However, because the psoriasis-like dermatitis observed in SKG mice is only slightly increased compared to wt (Figure 1C), yet SKG mice have much higher ROS levels on day 7 and 11, the difference is likely attributable to arthritis onset. Notably, a
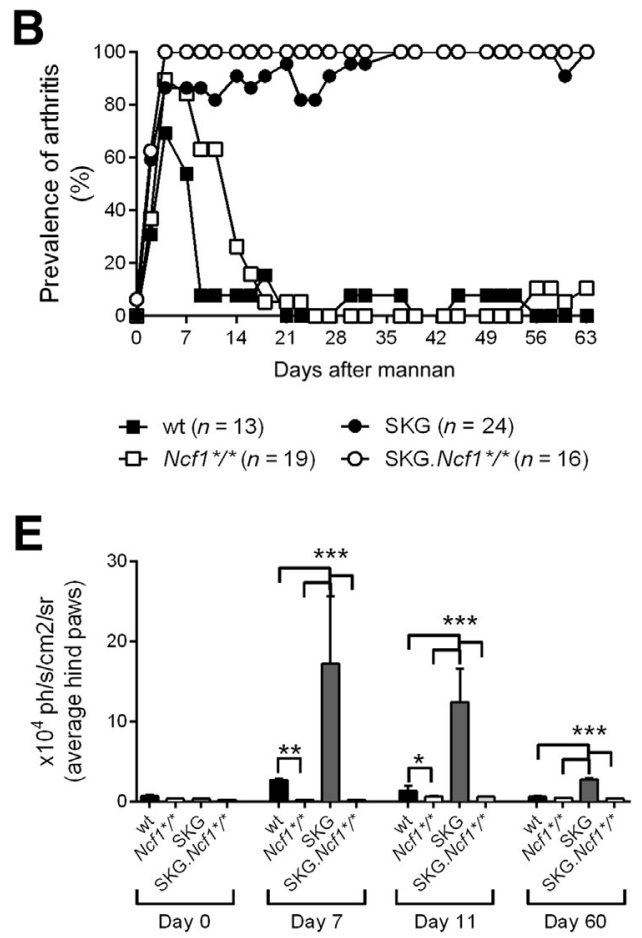

Figure 1 Reactive oxygen species (ROS) deficiency caused by a defective NADPH oxidase 2 complex worsens autoimmunity in SKG mice. Autoimmunity was induced with $20 \mathrm{mg}$ of mannan in wt, Ncf1*/* , SKG, and SKG.Ncf1*/* mice. Mice were clinically assessed using a 60 -point scoring system for joint inflammation (A) using a threshold of 2 to assess prevalence of arthritis (B). Skin inflammation was measured using a 3-point scoring system (C) using a threshold of 1 to assess prevalence of psoriasis-like dermatitis (D). E: Concentrations of ROS in joints were measured in vivo using the bioluminescent probe L-012 and are presented as the average bioluminescent signal of both hind paws. Results were analyzed using two-way analysis of variance with Bonferroni post hoc test (A and $\mathbf{C}$ ) and one-way analysis of variance with Tukey post hoc test (E). $n=2$ to $13(\mathbf{E}) .{ }^{*} P<0.05,{ }^{*} P<0.01$, and ${ }^{* * *} P<0.001$. 


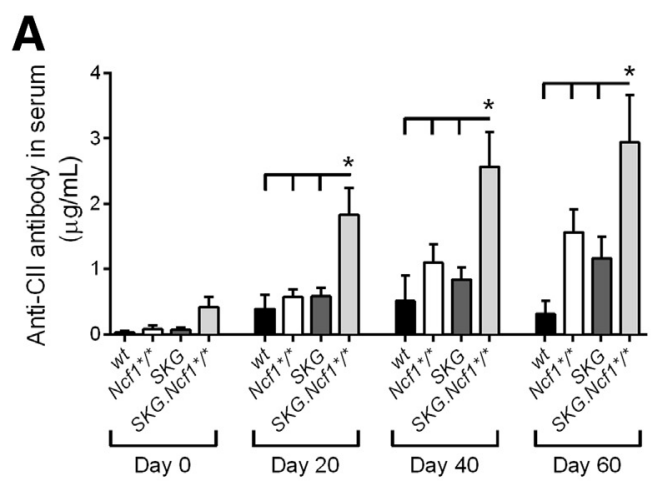

B

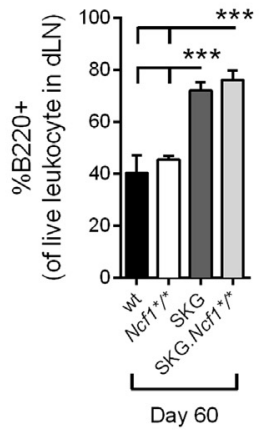

C

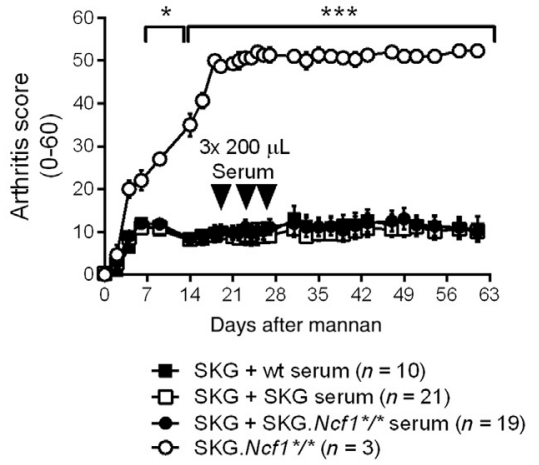

Figure 2 Reactive oxygen species (ROS)-deficient SKG mice have an increased collagen type II (CII) antibody response. A: Antibody concentrations in sera recognizing CII were measured before (day 0 ) and after mannan injection (days 20, 40, and 60). B: Frequency of B cells in joint draining lymph nodes 60 days after mannan injection determined by flow cytometry using B220 as marker. C: SKG mice were injected i.p. with 20 mg of mannan. At days 19, 23, and 27, these mice were injected with $200 \mu \mathrm{L}$ i.v. of serum from wt, SKG, or SKG.Ncf1*/* collected 60 days after mannan injection. Data were pooled respectively from three $(\mathbf{A}$ and $\mathbf{B})$ and two $(\mathbf{C})$ separate experiments with similar outcomes to increase statistical power. Results were analyzed using one-way analysis of variance with Tukey post hoc test $(\mathbf{A}$ and $\mathbf{B})$ and two-way analysis of variance with Bonferroni post hoc test $(\mathbf{C}) . n=4$ to $27(\mathbf{A}$ and $\mathbf{B})$. ${ }^{*} P<0.05,{ }^{* * *} P<0.001$.

high spike in ROS that lasts only a few days was observed in SKG mice during disease onset (Supplemental Figure S1). After this peak, ROS concentrations were drastically reduced but remained higher than baseline level once the arthritis was established. The impact of this ROS peak, which is likely to be a result of rapid migration of ROS-producing cells such as neutrophils and macrophages into joints, remains unclear. Taken together, ROS deficiency in SKG mice have the greatest impact on disease severity in the chronic phase. This might be because of a higher response of adaptive immunity, which takes longer time to fully develop, but still could be influenced by ROSproducing cells in the acute phase.

\section{ROS-Deficient SKG Mice Have an Increased Collagen Type II Ab Response}

SKG mice were previously shown to develop a spontaneous antibody $(\mathrm{Ab})$ response toward joint-specific self-antigens, such as CII. ${ }^{17}$ We found that ROS-deficient SKG.Ncf1 ${ }^{* *}$
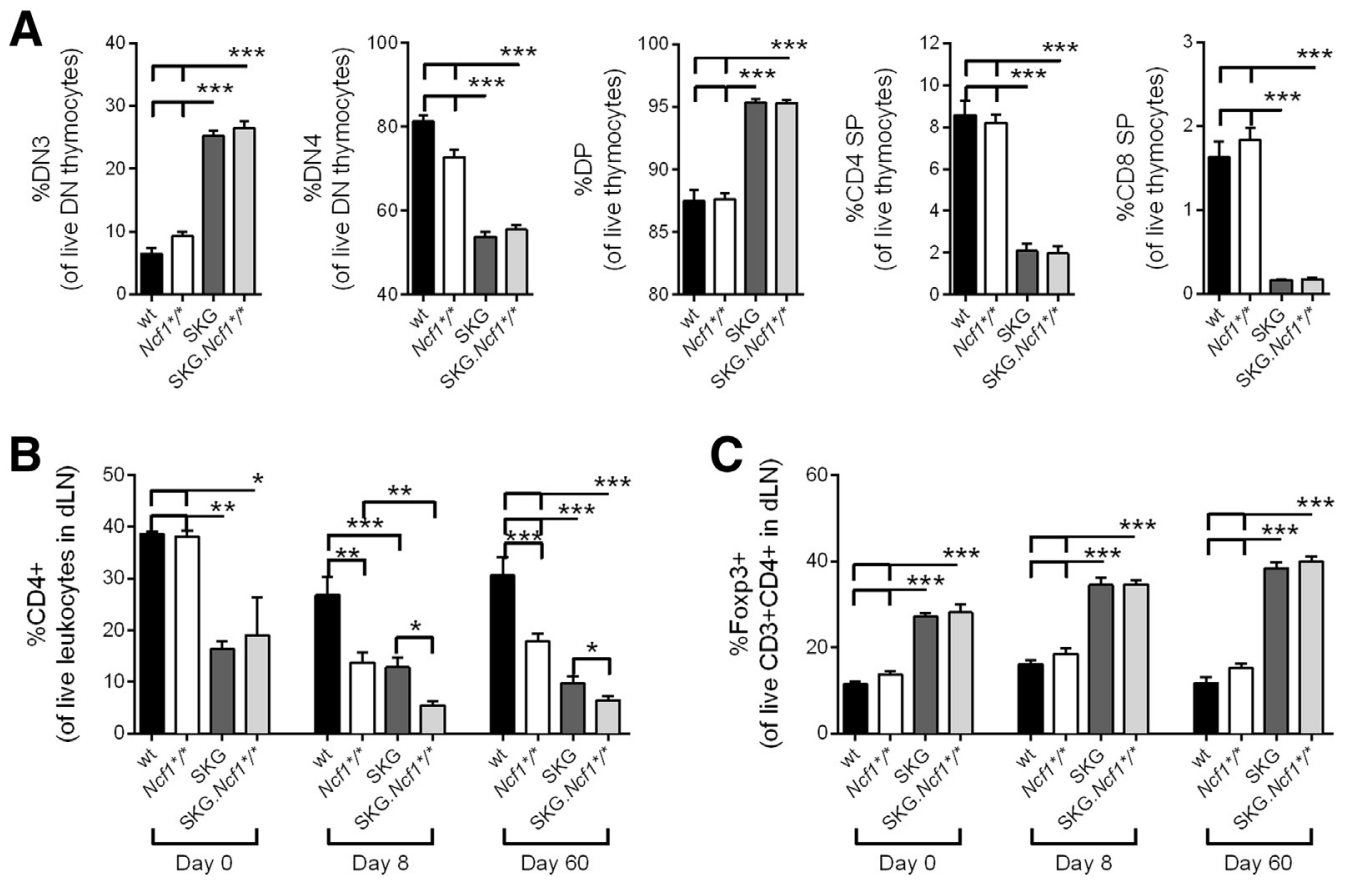

Figure 3 Reactive oxygen species (ROS) deficiency does not affect thymocyte or peripheral T-cell numbers in SKG mice. A: Frequency of thymocyte maturation stages was determined in naïve 21-day-old wt, Ncf1 ${ }^{* / *}$, SKG, and SKG.Ncf1*/* mice by flow cytometry. Frequency of CD4 T cells (B) and Foxp3 expression in $\mathrm{CD}^{+} \mathrm{CD}^{+}$cells (C) from joint draining lymph nodes 0,8 , and 60 days after mannan injection. Results were analyzed using one-way analysis of variance with Tukey post hoc test. $n=5$ to $8(\mathbf{A}) ; n=2$ to 13 (B and $\mathbf{C}$ ). ${ }^{*} P<0.05,{ }^{*} P<0.01$, and ${ }^{* * *} P<0.001$. 
mice have enhanced joint-specific self-reactivity compared with SKG mice already 20 days after mannan injection (Figure 2A). Interestingly, $N c f 1^{* * *}$ mice had increased concentrations compared to wt $(P<0.05)$ at day 60 despite any signs of joint inflammation. The increase in anti-CII $\mathrm{Ab}$ levels in SKG.Ncf1 ${ }^{* * *}$ mice could not be explained by a mere increase in B cells as in the chronic phase of arthritis, at day 60 after mannan injection, the frequency of B220 cells in dLN was significantly increased in both SKG and SKG.Ncf1 ${ }^{* / *}$ mice (Figure 2B). Thus, ROS deficiency did not further increase this difference. Taken together, these results demonstrate an increased B cell response caused by the additive effects of the mutation in Ncfl and ZAP70.

To directly assess the potential pathological role of autoAbs in SKG.Ncf1 ${ }^{* * *}$ mice, regular SKG mice received mannan followed by repetitive i.v. injections of serum isolated from arthritic SKG. $N c f 1^{* * *}$ mice (Figure 2C). These mice were therefore akin to SKG mice with the exception that circulating Abs in their serum mimicked those of SKG. $N c f 1^{* * *}$. The presence of higher concentrations of selfreactive $\mathrm{Ab}$ in SKG.Ncf1 ${ }^{* / *}$ serum compared to either wt or SKG did not influence the arthritis severity on transfer; thus, no clear role of the spontaneously developed auto-Abs could be determined in this experimental setting.

\section{ROS Deficiency Does Not Affect Thymocyte or Peripheral T-Cell Numbers in SKG Mice}

The defect in thymic selection because of the ZAP70 mutation is central in the pathogenesis of arthritis in SKG mice. We therefore investigated if the lack of ROS affected the thymocyte selection in 21-day-old SKG.Ncf1 ${ }^{* * *}$ mice (Figure 3A). Despite the clear impact of the $s k g$ mutation in thymic selection for both the passage from the double negative 3 to double negative 4 stage, and from double positive to either CD4 or CD8 single positive cells, no difference was observed because of the mutation in Ncfl. The defective thymic output of mature T cells in SKG mice was reflected by a lower $\mathrm{CD} 4^{+} \mathrm{T}$-cell frequency in the periphery of naïve SKG and SKG.Ncf1 $1^{* * *}$ mice (Figure 3B). However, after mannan injection, ROS deficiency significantly affected circulating $\mathrm{T}$ cells, resulting in a reduction in $\mathrm{CD}^{+}$T-cell frequency in dLN of SKG. $N$ cf $1^{* * *}$ mice compared to SKG mice, both after 8 and 60 days. This reduction can be mainly attributed to an increase of neutrophils and macrophages in dLN of ROS-deficient mice. Notably, throughout the disease course, the frequency of $\mathrm{CD}^{+}{ }^{+} \mathrm{Foxp}^{+}{ }^{+}$regulatory $\mathrm{T}$ cells was only affected by the $s k g$ mutation and not by ROS deficiency (Figure 3C).
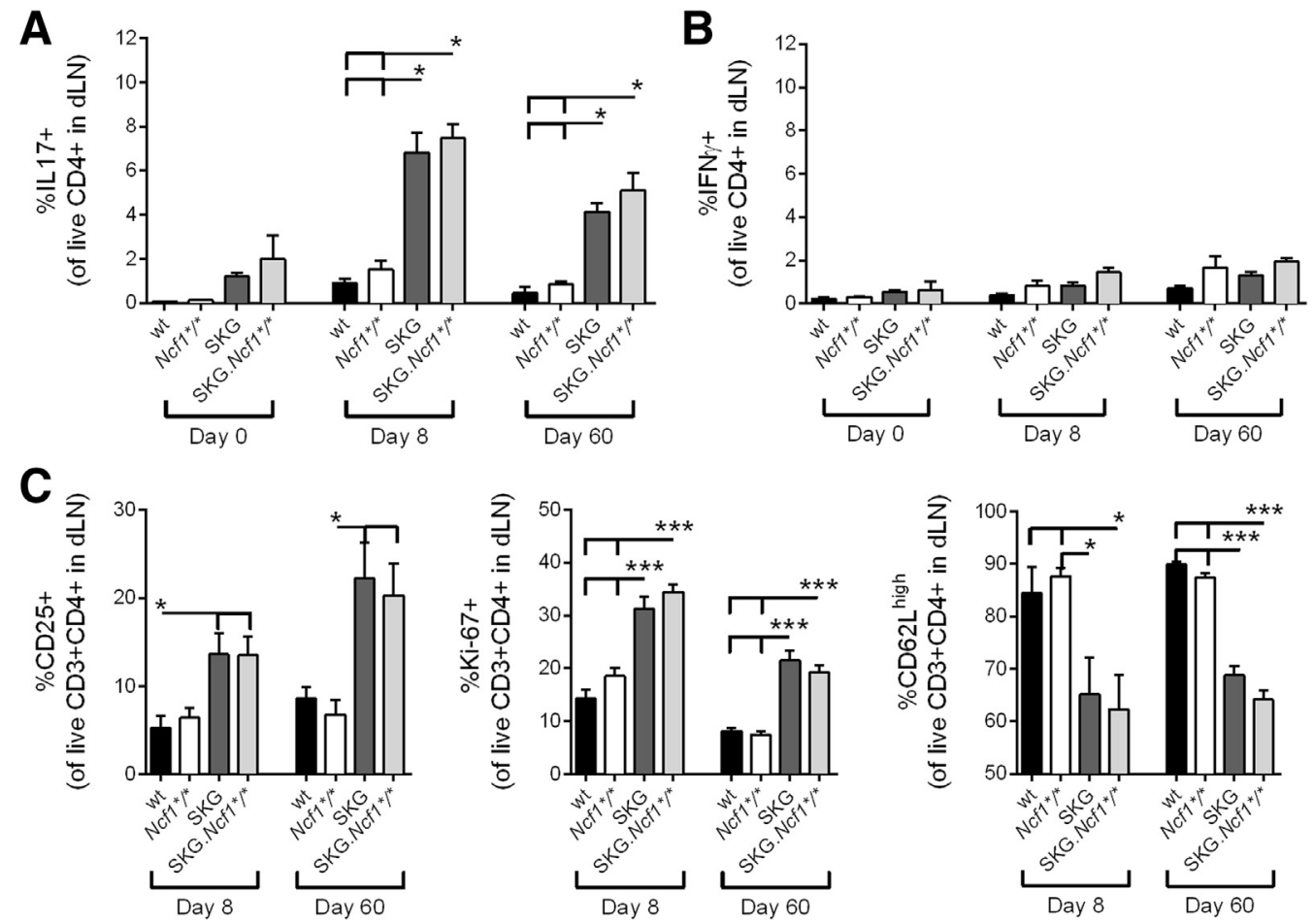

Figure 4 The T-cell-activation profile is mainly attributed to the mutation in ZAP70 and not in Ncf1. Joint draining lymph nodes were collected at days 0,8 , and 60 after mannan injection. Cells were stimulated in vitro with PMA/ionomycin and analyzed by flow cytometry to measure IL-17 (A) and interferon (IFN)- $\gamma$ (B) producing $\mathrm{CD}^{+} \mathrm{T}$ cells. C: Cells were also analyzed by flow cytometry to determine activation status in regards to CD25, Ki-67, and CD62L expression. Results were analyzed using one-way analysis of variance with Tukey post hoc test. Day 0: $n=2 \mathrm{wt}, n=5 \mathrm{Ncf1}{ }^{*}{ }^{*}, n=7 \mathrm{SKG}, n=4 \mathrm{SKG} . \mathrm{Ncf} 1^{*} /^{*} ;$ Day 8: $n=9 \mathrm{wt}, n=10$ Ncf1 ${ }^{*} /{ }^{*}, n=12$ SKG, $n=12$ SKG.Ncf1* ${ }^{*} ;$ Day $60: n=7$ wt, $n=12 \mathrm{Ncf1} 1^{*} /{ }^{*}, n=18$ SKG,$n=13$ SKG.Ncf1 ${ }^{*}{ }^{*} .{ }^{*} P<0.05,{ }^{* * *} P<0.001$. PMA, phorbol $12-$ myristate 13-acetate. 
The T-Cell-Activation Profile Is Mainly Attributed to the Mutation in ZAP70 and Not in Ncf1

$\mathrm{T}$ helper 17 cells have been shown to have a critical role in SKG arthritis, and we therefore further investigated if ROS deficiency affected T-cell activation and IL-17 production. ${ }^{20}$ Indeed, IL-17 production by $\mathrm{CD} 4^{+} \mathrm{T}$ cells is significantly increased in SKG mice after mannan exposure (Figure 4A). However, SKG.Ncf1 ${ }^{* * *}$ mice had similar levels of IL-17 positive $\mathrm{CD}^{+} \mathrm{T}$ cells despite higher arthritis severity (Figure 4A). Interestingly, there was no significant difference between groups in IFN $\gamma$ production by $\mathrm{CD}^{+} \mathrm{T}$ cells (Figure 4B). These results reinforce the idea that SKG arthritis is IL-17 dependent and IFN $\gamma$ independent. ${ }^{20,26}$ With regard to general T-cell activation, SKG mice had significantly increased levels of $\mathrm{CD} 25^{+}, \mathrm{CD} 62 \mathrm{~L}^{\text {low }} \mathrm{T}$ cells that also proliferated more according to Ki-67 expression (Figure 4C). However, the lack of ROS did not result in significant differences in this case either. In summary, the Tcell activation and differentiation profile appears to be primarily affected by the mutation in ZAP70 and not by the lack of ROS.

\section{ROS Deficiency in the Periphery Enhances Autoimmunity after Mannan Injection}

Although the ROS deficiency does not seem to block any step of thymic selection or to alter the activation status of $T$ cells in SKG mice, the T-cell repertoire per se could still be affected. To evaluate if the Ncfl mutation and the subsequent lack of ROS caused further break in central tolerance, we designed chimeric mice in which $\mathrm{T}$ cell repertoires generated in absence of ROS was transferred and tested in ROS-sufficient mice, and vice versa. T-cell deficient mice were therefore injected with peripheral cells isolated from $\mathrm{dLN}$ of SKG.Ncf1 ${ }^{* * *}$ or SKG mice.

At first, donor dLN cells isolated 5 days after mannan priming were transferred to test if those cells, taken at disease onset, were sufficiently activated to transfer autoimmunity in recipients. The level of $\mathrm{CD}^{+} \mathrm{CD}^{+}{ }^{+} \mathrm{T}$ cells in blood stabilized after 2 weeks and reached a concentration of approximately $50 \%$ of that of $\mathrm{T}$ cells in age-matched naïve SKG controls (Figure 5A). When no mice had developed arthritis or psoriasis-like dermatitis 3 weeks after transfer (data not shown), recipients were injected with mannan.
A

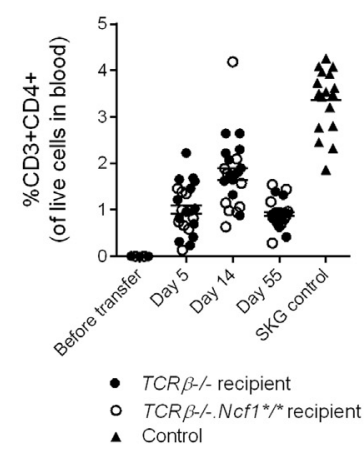

D

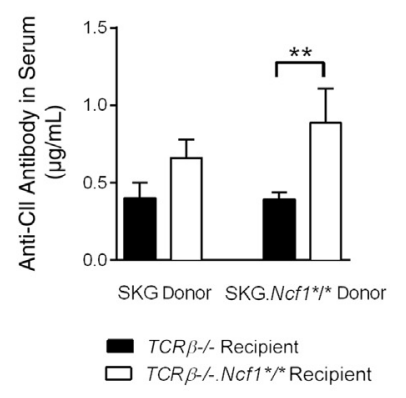

B

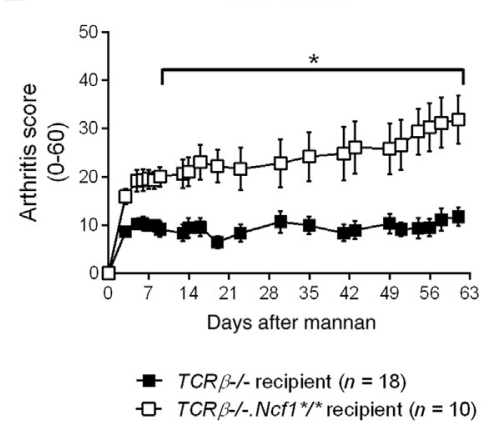

E

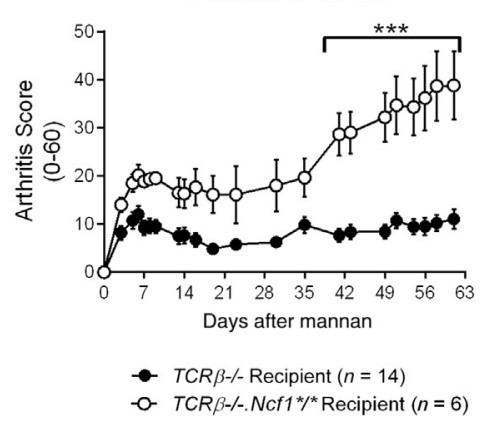

CKG donors

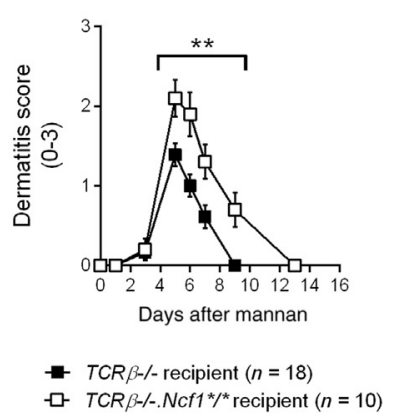

$\mathbf{F}$ SKG.NCf1*/* Donors

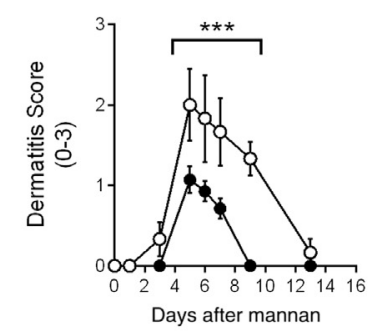

- TCR $\beta-/$ Recipient $(n=14)$

-o- TCR $\beta-/-. N c f 1^{* *}$ Recipient $(n=6)$

Figure 5 Reactive oxygen species (ROS) deficiency in the periphery enhances autoimmunity after mannan injection. Cells isolated from joint draining lymph nodes from SKG and SKG.Ncf1*/* mice primed 5 days earlier with $20 \mathrm{mg}$ of mannan were transferred to TCR $\beta^{-/-}$and TCR $\beta^{-/-}$. Ncf1 $1^{* / *}$ mice. Autoimmunity was then triggered with $20 \mathrm{mg}$ of mannan after 14 days of repopulation. A: Repopulation of $\mathrm{CD} 4^{+} \mathrm{T}$ cells in blood was monitored by flow cytometry. Chimeric mice transferred with cells from SKG (B and C) or SKG.Ncf1*/* (E and F) were clinically assessed using a 60-point scoring system for joint inflammation ( $\mathbf{B}$ and $\mathbf{E}$ ) and a 3-point scoring system for skin inflammation ( $\mathbf{C}$ and $\mathbf{F}$ ). D: Concentration of antibody recognizing collagen type II in serum of arthritic mice at day 60 . Data were pooled from two separate experiments with similar outcomes to increase statistical power. Results were analyzed using twoway analysis of variance with Bonferroni post hoc test. ${ }^{*} P<0.05,{ }^{*} P<0.01$, and ${ }^{* * *} P<0.001$. 
As opposed to $T C R \beta^{-/-}$mice, which were previously shown to develop transient joint and skin inflammation on mannan exposure, ${ }^{22}$ chimeric mice developed chronic arthritis mimicking SKG arthritis (Figure 5, B and E). This demonstrates that the transferred cells from SKG mice were essential to obtain chronic arthritis. Severity was overall lower in chimeric mice compared to regular SKG and SKG.N $c f 1^{* * *}$ controls, which might be explained by the lower number of $\mathrm{CD}^{+} \mathrm{T}$ cells in the chimeric mice. Nevertheless, the results clearly indicate that the source of transferred cells (SKG or SKG.Ncf1 ${ }^{* * *}$ mice) did not have a significant impact on disease severity, which suggests that the T-cell repertoire is not influenced in a major way by ROS deficiency in SKG mice. On the other hand, the lack of ROS in recipients clearly enhanced arthritis severity
(Figure 5, B and E). Moreover, psoriasis-like dermatitis was also unaffected by the source of transferred cells but depended on the lack of ROS in recipients (Figure 5, C and F). This was expected because the main cell populations transferred are $\alpha \beta T$ cells and B cells, and these cells are not essential for this pathology. ${ }^{22}$ Regarding B-cell response, it was higher in $T C R \beta^{-/-} . N c f 1^{* / *}$ compared to $T C R \beta^{-/-}$in mice. Indeed, there was an increased reactivity toward CII for mice receiving SKG.Ncfl ${ }^{* * *}$ cells $(P=0.0038)$ (Figure 5D) and a trend in mice receiving SKG cells $(P=0.11)$. Taken together, these results show that although SKG $\mathrm{T}$ cells are essential to develop chronic arthritis, it is not an intrinsic T-cell defect or a shifted repertoire because of ROS deficiency that enhanced autoimmunity, but rather peripheral lack of ROS during either T-

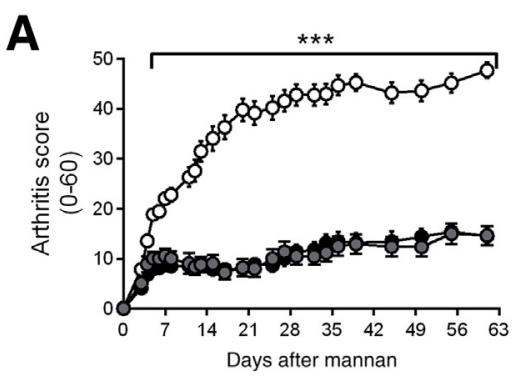

- SKG $(n=24)-0$ SKG.Ncf1*** $(n=26)-0$ SKG.MN $(n=17)$

C

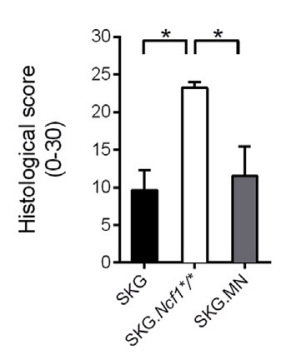

G

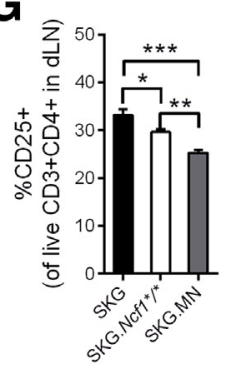

D
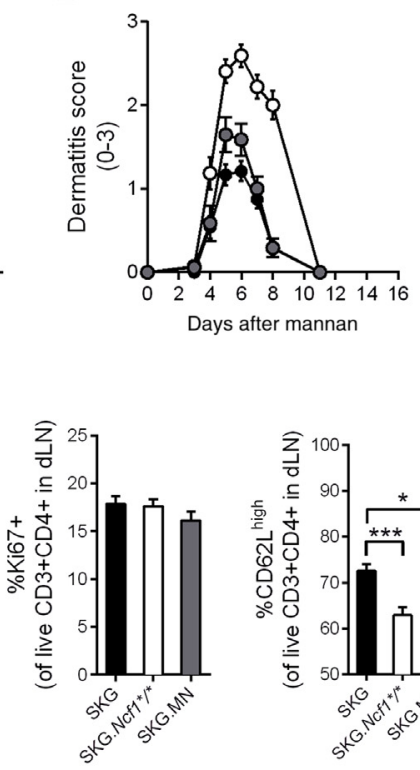

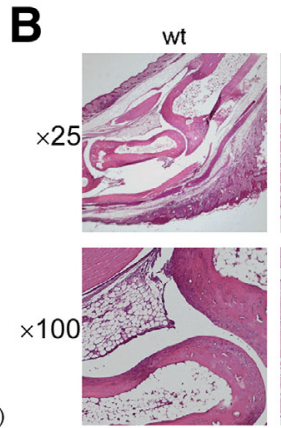

E

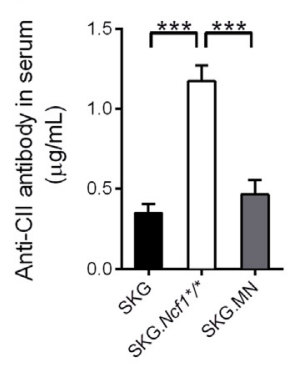

H

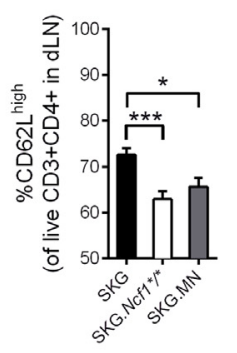

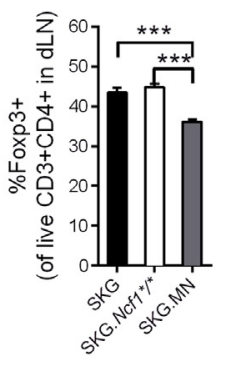

SKG
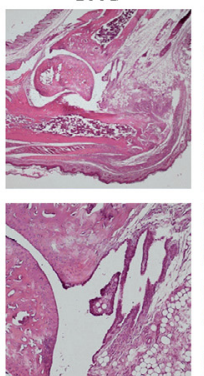

$\mathbf{F}$
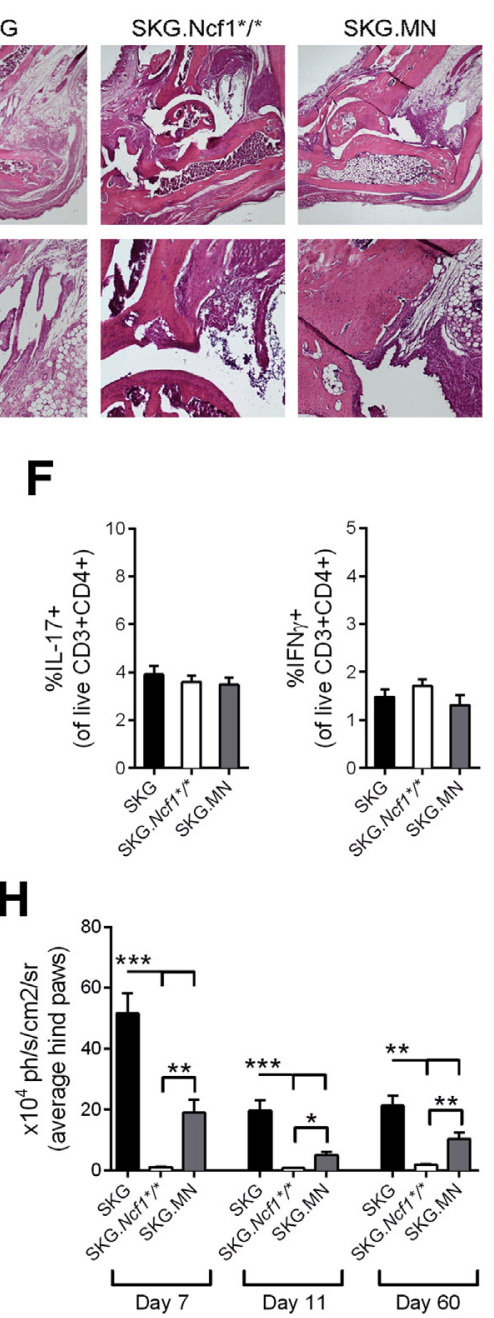

Figure 6 Macrophage-derived reactive oxygen species (ROS) protect against autoimmunity in SKG mice. Autoimmunity was triggered with 20 mg of mannan in SKG, SKG.Ncf1*/*, and SKG.MN. Arthritis was clinically assessed using a 60-point scoring system for joint inflammation (A) and by histology of hind paw joints stained with hematoxylin and eosin at day 60 (B) using a 30-point score (C). D: Psoriasis-like dermatitis was clinically evaluated using a 3-point scoring system for the skin phenotype. E: Antibody concentrations recognizing collagen type II were measured at day 60 . F: Joint draining lymph nodes were collected at day 60 and stimulated in vitro with PMA/ionomycin to measure IL-17- and interferon $\gamma$ (IFN $\gamma$ )-producing CD4 ${ }^{+}$T cells by flow cytometry. G: Lymph node cells were also analyzed by flow cytometry to determine activation status in vivo in regards to CD25, Ki-67, CD62L, and Foxp3 expression. H: R0S production in joints was compared at days 7, 11, and 60 using the bioluminescent probe L-012. Average bioluminescent signal of both hind paws were used for comparison. Data were pooled from three separate experiments with similar outcomes to increase statistical power. Results were analyzed using two-way analysis of variance with Bonferroni post hoc test. ${ }^{*} P<0.05,{ }^{* *} P<0.01$, and ${ }^{* * *} P<0.001$. PMA, phorbol 12-myristate 13-acetate. 
cell activation or tissue invasion by neutrophils and macrophages.

\section{Macrophage-Derived ROS Protects Against Autoimmunity in SKG Mice}

To investigate if ROS produced by phagocytes affect disease severity in ROS-deficient SKG mice, a transgene with functional Ncf1 under hCD68 promoter was introduced in SKG.Ncfl ${ }^{* / *}$ mice. These transgenic mice $(S K G . M N)$ are identical to their littermates SKG.Ncf1 ${ }^{* * *}$, except for the restored production of ROS by monocytes and macrophages only. Strikingly, macrophage-derived ROS had major impact on autoimmunity in SKG mice (Figure 6). Arthritis severity of both the acute and chronic arthritis phase in SKG.MN mice was reduced compared to SKG.Ncf1 ${ }^{* * *}$, and was similar to SKG mice (Figure 6A). This comparable severity between SKG and SKG.MN was further confirmed by histology (Figure 6, B and C). The severity of psoriasislike dermatitis in SKG.MN mice was similar to that of SKG mice, which in turn was significantly lower than SKG.Ncfl ${ }^{* * *}(P<0.001)$ (Figure 6D). Furthermore, antibody concentrations were also normalized by macrophagederived ROS (Figure 6E). T-cell cytokine production and activation marker expression revealed a proinflammatory state in SKG.MN, which was comparable to both SKG and SKG.Ncfl ${ }^{* / *}$ (Figure 6, F and G). Altogether, these results demonstrate that ROS originating from macrophages was sufficient to reduce disease severity to the levels observed in fully ROS sufficient SKG mice.

Interestingly, although most phenotypes were restored by macrophage-derived ROS, local concentrations of ROS in arthritic joints were not fully restored (Figure 6H). Indeed, SKG.MN mice had detectable ROS in the acute and chronic phase $\left(P<0.01\right.$ compared with SKG.Ncfl $\left.{ }^{* * *}\right)$, which demonstrated that macrophages actively secrete ROS in joints throughout the whole disease. However, SKG mice, in which ROS can also be produced by neutrophils, had higher levels of ROS in both the acute and chronic phase. This suggests that activated neutrophils are present in joints of SKG mice and produce ROS throughout the disease. However, the neutrophil-derived ROS do not seem to affect disease severity because SKG and SKG.MN mice have different levels of ROS in joints but similar disease. Thus, ROS derived from neutrophils do not seem to have any impact on SKG arthritis as long as SKG mice have at least macrophages with a capacity to produce ROS.

\section{Discussion}

ROS deficiency enhances severity of both arthritis and psoriasis-like dermatitis in SKG mice. A major conclusion from this study is that a restored ROS burst capacity in macrophages has a dramatic effect on disease severity in these mice. This supports previous results from T-cell independent models such as psoriasis-like dermatitis and psoriasis-arthritis, but also T-cell-dependent models such as CIA. ${ }^{14}$ However, as opposed to CIA, arthritis in SKG mice is enhanced by ROS independently of further T-cell activation or skewing toward $\mathrm{T}$ helper 17 differentiation. ${ }^{11,12}$ Moreover, we could not detect increased arthritogenicity of $\mathrm{T}$ cells because of ROS deficiency by adoptive transfer, which suggests that the break in T-cell tolerance toward endogenous CII observed in CIA is more likely to be related to a peripheral effect. ${ }^{13}$

The autoimmunity that follows mannan exposure in SKG mice can be divided in two phases: an onset/acute phase (day 0 to 10) and a chronic phase (after day 10). This distinction is vital to decipher how ROS deficiency is most likely to enhance autoimmunity. The acute phase comprises both arthritis and psoriasis-like dermatitis, and was shown to be IL-17 dependent yet $\alpha \beta$ T-cell independent in non-SKG mice. ${ }^{22}$ It was further concluded that the cellular source of IL-17 in non-SKG mice is likely $\gamma \delta \mathrm{T}$ cells. However, on the basis of literature and our data (Supplemental Figure S2), $\gamma \delta \mathrm{T}$ cells are unlikely to be the main source in SKG mice after mannan injection because IL-17 production is drastically reduced in their $\gamma \delta$ T-cell population. ${ }^{27}$ On the other hand, the chronic phase is restricted to arthritis and was shown to be driven by IL-17 from CD4 ${ }^{+} \mathrm{T}$ cells. ${ }^{20}$ Because the lack of ROS affects both acute and chronic immunity, we can conclude that it either affects a pathway common to both phases, or it affects several steps of the inflammatory cascade.

Regardless of its source, IL-17 is a common denominator of both acute and chronic inflammation in SKG mice. IL-17 is important in the recruitment, activation, and migration of neutrophils. ${ }^{28,29}$ Neutrophils are essential in the acute phase, and are found in high number in chronically inflamed joints of SKG mice (Supplemental Figure S3). ${ }^{22}$ ROS deficiency further increased the amount of neutrophils in synovia, which suggest that local ROS concentration could directly or indirectly influence neutrophil migration or survival. These neutrophils, despite lack of ROS, are still capable of promoting tissue destruction (eg, by the release of degradative enzymes and the modulation of inflammation) and could be implicated in enhancing severity of autoimmunity. ${ }^{30}$

Consistent with this, our results show that ROS produced by macrophages reduced disease severity. Local secretion of ROS by macrophages could be directly responsible for limiting survival of neutrophils in inflamed tissue, although it is unclear if it could also rescue neutrophil extracellular trap formation. Alternatively, local ROS could induce M2 differentiation of macrophage and thus limit inflammation, whereas ROS deficiency could favor M1 phenotype and enhance ongoing inflammation. ${ }^{31}$ Although ROS restored specifically in macrophages reverted this phenomena, we cannot exclude that ROS restored in neutrophils could have a similar effect. This would alter the interpretation of several findings, such as the priming of neutrophils to produce more ROS by psoriatic keratinocytes, which could be a negative 
feedback mechanism meant to limit tissue damage in chronic inflammation. ${ }^{32}$

SKG mice are known to spontaneously produce jointspecific antibodies. Similar to previous attempts, our experiments with serum transfer do not show that the humoral response in SKG mice plays a significant role for arthritis. In fact, SKG mice that lack B cells $\left(\mu M T^{-/}\right)$do not significantly differ in disease severity compared with regular SKG mice (Supplemental Figure S4). ${ }^{17}$ Nevertheless, this does not exclude a pathological role of enhanced humoral response in ROS-deficient mice. Despite being increased by ROS deficiency, the absolute concentrations of anti-CII Ab remained a thousandfold lower compared with concentrations in CIA or collagen antibody-induced arthritis. ${ }^{21,33}$ However, although we chose to study the CII response because it is joint specific, the disease-related B cell antigens are likely to be several different proteins. ${ }^{34}$ The serum transfer experiments, which do not indicate a high degree of pathogenicity, take the diverse Ab response in SKG.Ncf1 $1^{\text {*/* }}$ mice into account. Still, we cannot exclude that higher quantities of serum may increase arthritis severity.

\section{Conclusion}

Several key questions remain as to how ROS affects autoimmunity. Nevertheless, our results clearly indicate a direct protective role of macrophage-derived ROS in SKG mice. Enhanced autoimmunity in absence of ROS cannot be explained by enhanced humoral responses; it does not result from T-cell selection or activation either. Whether inducing supraphysiological ROS production could revert ongoing autoimmunity remains an open question, which would provide valuable information on anti-inflammatory mechanisms of ROS and would be of high relevance in the perspective of controlling inflammatory disorders.

\section{Acknowledgments}

We thank Shimon Sakaguchi (Kyoto University, Kyoto, Japan) for the kind gift of ZAP70 ${ }^{\text {skg/skg }}$ mice; Carlos Palestro, Kristina Palestro, and Evelina Wernersson for animal care; and Emma Mondoc for help with joint histology.

\section{Supplemental Data}

Supplemental material for this article can be found at http://dx.doi.org/10.1016/j.ajpath.2016.05.014.

\section{References}

1. Fang FC: Antimicrobial reactive oxygen and nitrogen species: concepts and controversies. Nat Rev Microbiol 2004, 2:820-832

2. Winkelstein JA, Marino MC, Johnston RB Jr, Boyle J, Curnutte J, Gallin JI, Malech HL, Holland SM, Ochs H, Quie P, Buckley RH, Foster CB, Chanock SJ, Dickler H: Chronic granulomatous disease: report on a national registry of 368 patients. Medicine 2000, 79: 155-169

3. Afonso V, Champy R, Mitrovic D, Collin P, Lomri A: Reactive oxygen species and superoxide dismutases: role in joint diseases. Joint Bone Spine 2007, 74:324-329

4. Kawai Y, Kubota E, Okabe E: Reactive oxygen species participation in experimentally induced arthritis of the temporomandibular joint in rats. J Dent Res 2000, 79:1489-1495

5. Simon HU, Haj-Yehia A, Levi-Schaffer F: Role of reactive oxygen species (ROS) in apoptosis induction. Apoptosis 2000, 5:415-418

6. Wing K, Klocke K, Samuelsson A, Holmdahl R: Germ-free mice deficient of reactive oxygen species have increased arthritis susceptibility. Eur J Immunol 2015, 45:1348-1353

7. De Ravin SS, Naumann N, Cowen EW, Friend J, Hilligoss D, Marquesen M, Balow JE, Barron KS, Turner ML, Gallin JI, Malech HL: Chronic granulomatous disease as a risk factor for autoimmune disease. J Allergy Clin Immunol 2008, 122:1097-1103

8. Olofsson P, Holmberg J, Tordsson J, Lu S, Akerstrom B, Holmdahl R: Positional identification of Ncf1 as a gene that regulates arthritis severity in rats. Nat Genet 2003, 33:25-32

9. Hultqvist M, Olofsson P, Holmberg J, Backstrom BT, Tordsson J, Holmdahl R: Enhanced autoimmunity, arthritis, and encephalomyelitis in mice with a reduced oxidative burst due to a mutation in the Ncf1 gene. Proc Natl Acad Sci U S A 2004, 101:12646-12651

10. van de Loo FA, Bennink MB, Arntz OJ, Smeets RL, Lubberts E, Joosten LA, van Lent PL, Coenen-de Roo CJ, Cuzzocrea S, Segal BH, Holland SM, van den Berg WB: Deficiency of NADPH oxidase components $\mathrm{p} 47$ phox and gp91phox caused granulomatous synovitis and increased connective tissue destruction in experimental arthritis models. Am J Pathol 2003, 163:1525-1537

11. Gelderman KA, Hultqvist M, Holmberg J, Olofsson P, Holmdahl R: T cell surface redox levels determine $\mathrm{T}$ cell reactivity and arthritis susceptibility. Proc Natl Acad Sci U S A 2006, 103:12831-12836

12. George-Chandy A, Nordstrom I, Nygren E, Jonsson IM, Postigo J, Collins LV, Eriksson K: Th17 development and autoimmune arthritis in the absence of reactive oxygen species. Eur J Immunol 2008, 38: 1118-1126

13. Hultqvist M, Backlund J, Bauer K, Gelderman KA, Holmdahl R: Lack of reactive oxygen species breaks $\mathrm{T}$ cell tolerance to collagen type II and allows development of arthritis in mice. J Immunol 2007, 179: $1431-1437$

14. Gelderman KA, Hultqvist M, Pizzolla A, Zhao M, Nandakumar KS, Mattsson R, Holmdahl R: Macrophages suppress T cell responses and arthritis development in mice by producing reactive oxygen species. $\mathrm{J}$ Clin Invest 2007, 117:3020-3028

15. Holmdahl R, Sareila O, Pizzolla A, Winter S, Hagert C, Jaakkola N, Kelkka T, Olsson LM, Wing K, Backdahl L: Hydrogen peroxide as an immunological transmitter regulating autoreactive T cells. Antioxid Redox Signal 2013, 18:1463-1474

16. Schauer C, Janko C, Munoz LE, Zhao Y, Kienhofer D, Frey B, Lell M, Manger B, Rech J, Naschberger E, Holmdahl R, Krenn V, Harrer T, Jeremic I, Bilyy R, Schett G, Hoffmann M, Herrmann M: Aggregated neutrophil extracellular traps limit inflammation by degrading cytokines and chemokines. Nat Med 2014, 20:511-517

17. Sakaguchi N, Takahashi T, Hata H, Nomura T, Tagami $T$, Yamazaki S, Sakihama T, Matsutani T, Negishi I, Nakatsuru S, Sakaguchi S: Altered thymic T-cell selection due to a mutation of the ZAP-70 gene causes autoimmune arthritis in mice. Nature 2003, 426:454-460

18. Hashimoto M, Hirota K, Yoshitomi H, Maeda S, Teradaira S, Akizuki S, Prieto-Martin P, Nomura T, Sakaguchi N, Kohl J, Heyman B, Takahashi M, Fujita T, Mimori T, Sakaguchi S: Complement drives Th17 cell differentiation and triggers autoimmune arthritis. J Exp Med 2010, 207:1135-1143

19. Yoshitomi H, Sakaguchi N, Kobayashi K, Brown GD, Tagami T, Sakihama T, Hirota K, Tanaka S, Nomura T, Miki I, Gordon S, Akira S, Nakamura T, Sakaguchi S: A role for fungal \{beta\}-glucans 
and their receptor Dectin-1 in the induction of autoimmune arthritis in genetically susceptible mice. J Exp Med 2005, 201:949-960

20. Hirota K, Hashimoto M, Yoshitomi H, Tanaka S, Nomura T, Yamaguchi T, Iwakura Y, Sakaguchi N, Sakaguchi S: T cell self-reactivity forms a cytokine milieu for spontaneous development of IL-17+ Th cells that cause autoimmune arthritis. J Exp Med 2007, 204:41-47

21. Bajtner E, Nandakumar KS, Engstrom A, Holmdahl R: Chronic development of collagen-induced arthritis is associated with arthritogenic antibodies against specific epitopes on type II collagen. Arthritis Res Ther 2005, 7:R1148-R1157

22. Khmaladze I, Kelkka T, Guerard S, Wing K, Pizzolla A, Saxena A, Lundqvist K, Holmdahl M, Nandakumar KS, Holmdahl R: Mannan induces ROS-regulated, IL-17A-dependent psoriasis arthritis-like disease in mice. Proc Natl Acad Sci U S A 2014, 111:E3669-E3678

23. Kelkka T, Hultqvist M, Nandakumar KS, Holmdahl R: Enhancement of antibody-induced arthritis via Toll-like receptor 2 stimulation is regulated by granulocyte reactive oxygen species. Am J Pathol 2012, 181:141-150

24. Burkhardt H, Koller T, Engstrom A, Nandakumar KS, Turnay J, Kraetsch HG, Kalden JR, Holmdahl R: Epitope-specific recognition of type II collagen by rheumatoid arthritis antibodies is shared with recognition by antibodies that are arthritogenic in collagen-induced arthritis in the mouse. Arthritis Rheum 2002, 46:2339-2348

25. Guerard S, Boieri M, Hultqvist M, Holmdahl R, Wing K: The SKG mutation in ZAP-70 also confers arthritis susceptibility in C57 Black mouse strains. Scand J Immunol 2016, 84:3-11

26. Hata H, Sakaguchi N, Yoshitomi H, Iwakura Y, Sekikawa K, Azuma Y, Kanai C, Moriizumi E, Nomura T, Nakamura T, Sakaguchi S: Distinct contribution of IL-6, TNF-alpha, IL-1, and IL-10 to T cell-mediated spontaneous autoimmune arthritis in mice. J Clin Invest 2004, 114:582-588

27. Wencker M, Turchinovich G, Di Marco Barros R, Deban L, Jandke A, Cope A, Hayday AC: Innate-like T cells straddle innate and adaptive immunity by altering antigen-receptor responsiveness. Nat Immunol 2014, 15:80-87

28. Korn T, Bettelli E, Oukka M, Kuchroo VK: IL-17 and Th17 cells. Annu Rev Immunol 2009, 27:485-517

29. Cua DJ, Tato CM: Innate IL-17-producing cells: the sentinels of the immune system. Nat Rev Immunol 2010, 10:479-489

30. Wright HL, Moots RJ, Edwards SW: The multifactorial role of neutrophils in rheumatoid arthritis. Nat Rev Rheumatol 2014, 10: 593-601

31. Zhang Y, Choksi S, Chen K, Pobezinskaya Y, Linnoila I, Liu ZG: ROS play a critical role in the differentiation of alternatively activated macrophages and the occurrence of tumor-associated macrophages. Cell Res 2013, 23:898-914

32. Guerard S, Allaeys I, Martin G, Pouliot R, Poubelle PE: Psoriatic keratinocytes prime neutrophils for an overproduction of superoxide anions. Arch Dermatol Res 2013, 305:879-889

33. Nandakumar KS, Svensson L, Holmdahl R: Collagen type II-specific monoclonal antibody-induced arthritis in mice: description of the disease and the influence of age, sex, and genes. Am J Pathol 2003, 163: $1827-1837$

34. Ito Y, Hashimoto M, Hirota K, Ohkura N, Morikawa H, Nishikawa H, Tanaka A, Furu M, Ito H, Fujii T, Nomura T, Yamazaki S, Morita A, Vignali DA, Kappler JW, Matsuda S, Mimori T, Sakaguchi N, Sakaguchi S: Detection of $\mathrm{T}$ cell responses to a ubiquitous cellular protein in autoimmune disease. Science 2014, 346:363-368 